\title{
Complicaciones en la artroscopía posterior de tobillo por portales clásicos y sin distracción
}

\author{
Complications in posterior ankle arthroscopy by classic and non-distraction portals \\ Yáñez-Arauz JM,* Raimondi N, ${ }^{\star}$ Tomé C, ${ }^{\S}$ Posse R,` Yáñez-Arauz S\| \\ Hospital Universitario Austral, Buenos Aires, Argentina.
}

RESUMEN. Introducción: La artroscopía posterior de tobillo permite el diagnóstico y tratamiento de múltiples patologías a través de un mínimo trauma. Los portales posteriores, asociados o no a distracción mecánica, permiten una buena visión de los tendones de la región y articulación substragalina. Objetivo: del presente estudio es evaluar las complicaciones intraoperatorias y postoperatorias de la artroscopía posterior del tobillo y retropié sin distracción articular y compararlas con las descritas en la bibliografía internacional. Material y métodos: Estudio retrospectivo que evaluó 49 pacientes sometidos a artroscopía posterior de tobillo por diversas patologías. Todos operados por un único cirujano, en dos instituciones de salud, durante un período de siete años. Se analizaron los resultados mediante la escala visual analógica (EVA) y la escala de la American Orthopaedic Foot and Ankle Society (AOFAS, por sus siglas en inglés). Se consignaron las complicaciones intra- y postoperatorias. El seguimiento promedio fue de 28 meses. Resultados: 33 varones y 16 mujeres (edad promedio 38.6 años). Hubo siete complicaciones (14.3\%): cuatro parestesias y/o hipoestesia del nervio sural, dos casos de dolor residual en un portal y un caso de dehiscencia de cicatriz. Mejoría promedio de EVA de 5.53 puntos y mejoría promedio del AOFAS de 35.78 puntos.
ABSTRACT. Introduction: Posterior ankle arthroscopy allows the diagnosis and treatment of multiple pathologies through minimal trauma. The use of posterior portals associated or not to mechanical distraction, allows a good vision of the posterior ankle, tendons of the region, and subtalar joint. Objectives: We evaluated intra- and postoperative complications of posterior ankle arthroscopy without joint distraction, and compared them with those described in international literature. Material and methods: A retrospective study that evaluated 49 patients undergoing posterior ankle arthroscopy for several pathologies. All of them operated by a single surgeon, in two health institutions, for seven years. Results: were analyzed using the visual analog scale and the AOFAS score. Intra- and postoperative complications were recorded. The average follow-up was 28 months. Results: 33 men and 16 women. The average age was 38.6 years old. There were seven complications (14.3\%): paresthesia and - or hypoesthesia of the sural nerve (four cases), residual pain in the portal (two cases), and portal scar dehiscence (one patient). The average improvement of VAS was 5.53 points, and the improved AOFAS score was an average of 35.78 points. Conclusions: Posterior ankle arthroscopy without distraction through the

\section{Nivel de evidencia: IV (serie de casos)}

* Medical Doctor. Jefe del Sector Pierna, Tobillo y Pie. Hospital Universitario Austral. Magister in Orthopaedic Surgery,

Universidad de Buenos Aires (UBA). Professor, Faculty of Medicine, Austral University.

* Staff del Sector Pierna, Tobillo y Pie. Hospital Universitario Austral.

$\S$ Fellow del Sector Pierna, Tobillo y Pie. Hospital Universitario Austral.

" Médico residente del Servicio de Ortopedia y Traumatología. Hospital Universitario Austral.

" Estudiante de medicina. Facultad de Ciencias Biomédicas. Universidad Austral.

Buenos Aires, Argentina.

Correspondencia:

Juan Manuel Yáñez-Arauz

Gral. Urquiza Núm. 340, Acassuso, Buenos Aires. Argentina. PC: 1641.

E-mail: jmyanez@cas.austral.edu.ar

Recibido: 10-09-2020. Aceptado: 03-02-2021.

Citar como: Yáñez-Arauz JM, Raimondi N, Tomé C, Posse R, Yáñez-Arauz S. Complicaciones en la artroscopía posterior de tobillo por portales clásicos y sin distracción. Acta Ortop Mex. 2021; 35(1): 46-50. https://dx.doi.org/10.35366/100930

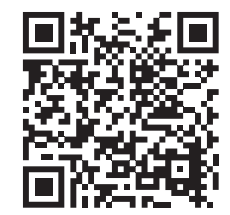


Conclusiones: La artroscopía posterior de tobillo sin distracción por medio de los clásicos portales posterolateral y posteromedial es una técnica segura, con un bajo índice de complicaciones mayores y una baja morbilidad para el paciente.

Palabras clave: Artroscopía de tobillo, artroscopía posterior, tobillo, complicaciones.

\section{Introducción}

La cirugía artroscópica del tobillo ha ido en constante crecimiento en los últimos 20 años. Hoy se considera un procedimiento más dentro de los que dispone el cirujano, que permite tanto realizar diagnósticos (no evidenciables por métodos de imágenes y el examen clínico), como tratar múltiples afecciones a través de una mínima agresión quirúrgica. Esto lleva a una menor morbilidad del paciente, un menor trauma de partes blandas y frecuentemente una más rápida recuperación funcional. ${ }^{1}$ Mediante la artroscopía posterior, es posible llegar a los distintos puntos articulares tanto de la cámara posterior del tobillo, como de la articulación subastragalina, sin necesidad de artrotomías ni osteotomías maleolares; a través de diversos portales posteriores nos permite acceder a procesos dolorosos del tobillo y del retropié, ${ }^{2}$ pudiendo visualizar, además de lo mencionado, los tendones retromaleolares y el Aquiles. Esto permite tratar sus patologías, fracturas de la región, síndromes friccionales posteriores, patología producida por os trigonum, lesiones osteocondrales, calcificaciones, fibrosis posteriores, remoción de cuerpos libres, etcétera. También con este método se puede realizar una artrodesis subastragalina y tratar patologías de esta articulación. ${ }^{3,4}$ Para realizar esta técnica de manera segura y minimizar riesgos, es fundamental conocer la anatomía artroscópica de la región posterior, muy bien descrita por distintos autores. ${ }^{5,6,7}$ Si bien diferentes estudios ${ }^{8,9}$ mencionan cómo realizar los portales y la seguridad de los mismos para no lesionar estructuras, como todo procedimiento quirúrgico puede presentar complicaciones. Existen múltiples publicaciones ${ }^{10,11,12,13,14,15}$ acerca de las complicaciones de la artroscopía anterior del tobillo, pero hay pocos artículos publicados que describan las complicaciones de la artroscopía posterior de tobillo sin distracción.

El objetivo del presente estudio fue evaluar las complicaciones intraoperatorias y postoperatorias de la artroscopía posterior del tobillo y retropié sin distracción articular y compararlas con las descritas en la bibliografía internacional.

\section{Material y métodos}

Se realizó un estudio retrospectivo en dos instituciones de salud de la provincia de Buenos Aires. Se incluyeron 49 pacientes intervenidos quirúrgicamente mediante una artroscopía posterior del tobillo entre Diciembre de 2011 y classic postero-lateral and posteromedial portals is a safe technique, with a low rate of complications.

Keywords: Ankle arthroscopy, posterior arthroscopy; ankle; complications.
Diciembre de 2018. Todas las artroscopías evaluadas se realizaron por los dos portales clásicos: posteromedial y posterolateral del tobillo descriptos por Van Dijk. ${ }^{9}$ No se utilizó ningún sistema de distracción (ni invasivo ni no invasivo) para la ejecución de la técnica. Se trabajó con manguito hemostático en el muslo. En todos los casos se utilizó una óptica de $4 \mathrm{~mm}$ a $30^{\circ}$ y el paciente se intervino en decúbito prono con el pie colgando de la mesa operatoria y un realce en la cara anterior del tercio distal de la tibia. Esto permitió la libre movilización del tobillo durante el procedimiento. El artroscopio junto a la óptica se introdujo siempre inicialmente por el portal lateral apuntando al segundo rayo, mientras que el shaver se introdujo en principio por el portal medial en dirección a la región lateral, a $90^{\circ}$ respecto a la óptica para evitar lesionar el paquete tibial. Ambos portales se realizaron para-aquileanos adyacentes al tendón. Se incidió la piel superficial y el ingreso al espacio posterior articular se realizó mediante instrumental romo. Todos los pacientes fueron operados por el mismo cirujano en ambos centros de atención. Esto se tuvo en cuenta para reducir las complicaciones debidas a la curva de aprendizaje. Siempre se trató de una cirugía ambulatoria, con una recuperación promedio de dos horas. Los pacientes fueron citados al primer control postoperatorio, a los siete días en promedio (entre seis y ocho días). Los diagnósticos previos a la cirugía fueron 14 pacientes con os trigonum doloroso, 14 pacientes con artrofibrosis posterior con síndrome de fricción posterior, siete tenosinovitis del flexor hallucis longus, cinco deformidades de Haglund con fibrosis posterior, tres lesiones osteocondrales astragalinas posteriores, tres artrodesis subastragalinas, una calcificación heterotópica periprotésica, una sinovitis vellonodular pigmentada y una fractura de maleolo posterior. El protocolo de estudio comprendió un completo examen preoperatorio con imágenes que confirmaron los diagnósticos precedentes antes de la intervención. No se efectuaron artroscopías diagnósticas en esta serie.

Se evaluaron las complicaciones intraoperatorias y postoperatorias. Los criterios de inclusión fueron: 1) pacientes sometidos a una artroscopía posterior de tobillo sin distracción articular; y 2) uso de dos únicos portales clásicos, posteromedial y posterolateral. Los criterios de exclusión fueron: 1) pacientes en quienes fue necesario recurrir a portales accesorios; y 2) aquellos casos en los que no se pudo realizar el seguimiento adecuado. El seguimiento consistió en controles periódicos y una historia clínica informatizada, consignando las complicaciones detectadas durante el 
procedimiento y en las consultas posteriores (anamnesis y examen físico), de manera que todo evento fue registrado en la historia clínica computarizada institucional. Se definió como complicación a «cada evento que surge como un problema adicional durante el procedimiento o después de este y es secundario a él». Asimismo, se citó a los sujetos para una evaluación final dentro del mayor plazo posible, según el caso, pero todos con un mínimo de 18 meses de postoperatorio. Para la evaluación del dolor se utilizó la escala visual analógica de 10 puntos, donde 0 fue sin dolor y 10 dolor intenso, evaluando tanto el preoperatorio como el postoperatorio a 18 meses. Se utilizó la escala de la American Orthopaedic Foot and Ankle Society (AOFAS por sus siglas en inglés) de tobillo y retropié para evaluar función, alineación y también dolor; en donde se registró la puntuación AOFAS en el preoperatorio y en el postoperatorio más alejada del seguimiento. El seguimiento promedio fue de 28 meses (rango 18-77).

Análisis estadístico: Los hallazgos fueron colocados en la historia clínica de forma estandarizada y en una planilla de cálculo. Se utilizó la media y los porcentajes para todas las variables cuantitativas en el estudio. Los datos obtenidos en el pre- y postoperatorio se compararon mediante la prueba t de Student para muestras con varianza desigual. Se consideró un valor estadísticamente significativo si la p era menor a 0.05 .

\section{Resultados}

Del total de artroscopías posteriores realizadas, $67.35 \%$ (33 pacientes) correspondió a varones y 32.65\% (16 pacientes) fueron mujeres. Se trataron 27 tobillos izquierdos y 22 derechos. El promedio de la edad fue de 38.6 años (rango 15-68 años). Se consignaron siete complicaciones (14.3\%) relacionadas con el procedimiento (Figura 1): cuatro casos de parestesia y/o hipoestesia del nervio sural (8.1\%) tratados con complejo vitamínico B1-B6-B12, todos mejoraron excepto

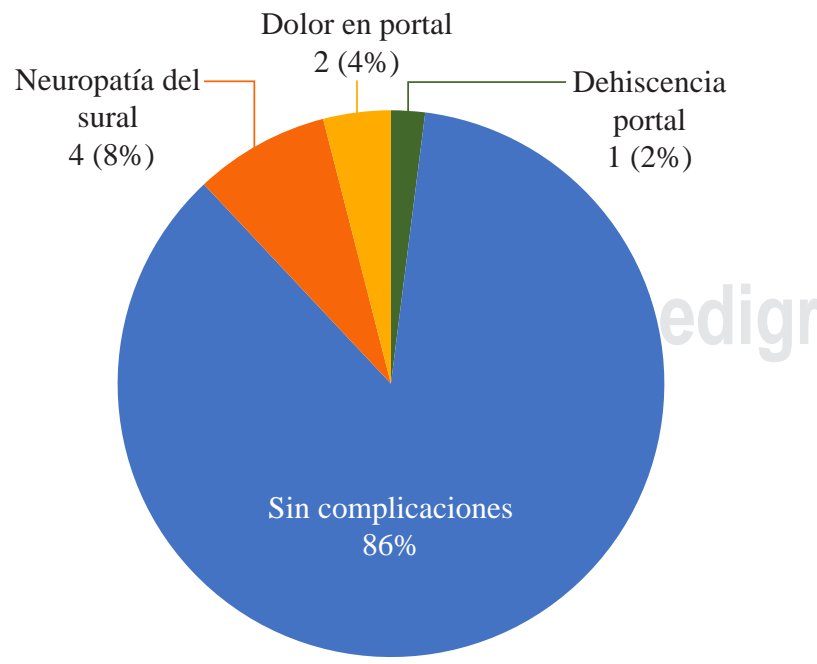

Figura 1: Complicaciones. un paciente que persistió con parestesias; dos casos de dolor en portal artroscópico (4\%) que se resolvió con infiltración con corticoides y fisioterapia; y un caso de dehiscencia del portal posterolateral (2\%) que cicatrizó mediante curaciones simples al mes de la cirugía. No se observaron infecciones superficiales ni profundas. Cinco pacientes refirieron dolor del tobillo en el postoperatorio sin signos de síndrome regional complejo, lo que consideramos como la historia evolutiva de su dolor previo a la cirugía asociado al trauma inflamatorio quirúrgico, por ello, no lo consignamos como complicación del procedimiento. La mejoría promedio según la escala AOFAS fue de 46.4 puntos promedio, con una media de 54.12 en el preoperatorio y de 89.9 puntos en el postoperatorio. El análisis comparativo entre el pre- y postoperatorio fue estadísticamente significativo, con un valor p: $<0.0005$. En cuanto a la escala visual analógica, se obtuvo un promedio de 7.96 en el preoperatorio y de 2.43 en el postoperatorio. El análisis comparativo arrojó una p: $<0.0005$ significativa.

\section{Discusión}

La artroscopía posterior del tobillo es un procedimiento que se volvió importante para el tratamiento de numerosas patologías articulares y extraarticulares del tobillo y subastragalina, con la ventaja de una recuperación más rápida y una hospitalización más corta comparada con las técnicas tradicionales abiertas. ${ }^{16}$ Sin embargo, existen pocos artículos que detallan las complicaciones de este procedimiento quirúrgico.

En relación a la lesión del nervio sural, Nickisch y colaboradores ${ }^{17}$ informan en su trabajo una prevalencia de $3.7 \%$ de lesiones neurológicas con afección del nervio sural en su mayoría. Abramowitz y su equipo ${ }^{18}$ informaron sobre la aparición de neuroapraxia del nervio sural en 19.5\% de sus pacientes. Noguchi y su grupo ${ }^{19}$ informaron que de 12 casos tratados mediante artroscopía posterior de tobillo, 8.33\% presentó una neuroapraxia del nervio sural y proponen la identificación roma del nervio para evitar su lesión. Para disminuir al mínimo el riesgo de lesiones neurológicas, Van Dijk y colaboradores ${ }^{9}$ recomiendan realizar el portal posterolateral al nivel o ligeramente por encima de la punta del maléolo lateral, adyacente al tendón de Aquiles. De esta manera, se minimiza el posible riesgo de lesión del nervio sural, que se describe cruzando el borde lateral del tendón de Aquiles en forma subcutánea, en promedio 9.8 cm (rango, 6.5 a $16 \mathrm{~cm}$ ) próximo a la inserción del tendón de Aquiles. ${ }^{20}$ Lee y su equipo ${ }^{21}$ describieron un portal cerca del borde lateral del tendón de Aquiles para reducir riesgos. En la serie presentada, utilizamos la técnica de portales descrita por Van Dijk y se tuvo especial cuidado en realizar la incisión de piel con una hoja No. 15 de bisturí (sólo la epidermis y dermis), realizando disección roma en el tejido celular subcutáneo (TCS) en sentido longitudinal al eje del miembro y en dirección al espacio interdigital entre el primer y segundo dedo del pie con una pinza Halsted mosquito, lo que evitaría la lesión inadvertida del nervio; a pesar 
de ello, fue la principal complicación observada en la serie (8.1\% de los casos). También es necesario mencionar que el intercambio repetitivo de instrumentos a través del portal y las maniobras de tracción del TCS con los mismos debe evitarse o reducirse, para no provocar la neuroapraxia del nervio sural que es superficial. Si bien la realización de portales accesorios posterolaterales podría generar mayor riesgo de lesión del nervio sural, en el presente trabajo no se incluyen pacientes con dicho portal.

Keeling y colaboradores ${ }^{22}$ describieron en un estudio cadavérico los riesgos de lesionar el paquete tibial posterior al intentar realizar una descompresión del tendón del flexor hallucis longus. Nickisch y su grupo ${ }^{17}$ mencionan en su trabajo cuatro lesiones del nervio tibial, dos de las cuales se resolvieron solas, una requirió liberación del túnel tarsiano y desbridamiento local resolviendo la clínica y la otra presentó una secuela neurológica a pesar de la neurólisis. Ellos sugieren que los pacientes con artritis, rigidez y deformidad postraumática del tobillo y/o retropié pueden tener un mayor riesgo de lesiones nerviosas durante la artroscopía posterior del tobillo. El portal posteromedial se debe hacer adyacente al borde medial del tendón de Aquiles, siguiendo las recomendaciones de Van Dijk y colaboradores ${ }^{9}$ y sólo seccionar la piel con la hoja de bisturí 15 . El ingreso se realiza por divulsión roma del TCS hacia lateral por delante del tendón de Aquiles, hasta tocar el trocar del artroscopio introducido por el portal posterolateral. De esa manera, se evita el riesgo de lesionar el paquete tibial posterior. Luego se va dirigiendo el shaver hacia anterior y medial hasta visualizar el tendón del flexor hallucis longus, lo que nos permite una muy buena visualización del tobillo y subtalar posterior. Hay que evitar ir más medial a dicho tendón por el riesgo neurovascular tibial posterior. ${ }^{23,24}$ En el presente trabajo, no hemos observado la lesión del nervio tibial, ni de su paquete vascular. Cabe mencionar que hay que tener presente y conocer las variaciones anatómicas de la región, que pueden aumentar el riesgo de lesión del nervio tibial, como por ejemplo el falso flexor hallucis longus. ${ }^{23}$

En relación con la posición del paciente en el acto quirúrgico, Lee y su grupo ${ }^{4}$ observaron que la realización de los portales posteriores es más difícil cuando el paciente está en posición supina o lateral, lo que puede aumentar el riesgo de lesiones neurovasculares.

En referencia a las infecciones, son poco frecuentes en la artroscopía posterior de tobillo. Dos casos (1.1\% de la muestra) fueron reportados por Nickisch y su equipo, ${ }^{17}$ necesitando uno de ellos tratamiento quirúrgico. En los casos de nuestra serie no hemos tenido complicaciones infecciosas superficiales ni profundas.

El síndrome regional complejo es menos frecuente que el que se produce en la artroscopía anterior de tobillo. ${ }^{13}$ Abramowitz y colaboradores ${ }^{18}$ reportaron un caso en su serie y Nickisch y su equipo ${ }^{17}$ mencionan dos casos que fueron tratados con fisioterapia y bloqueo simpático, mejorando dentro de los seis meses postoperatorios. En la serie presentada no hemos reportado casos de síndrome regional complejo.
Si bien se reporta engrosamiento con dolor y disminución de la movilidad del tendón de Aquiles, ${ }^{17}$ al usar portales transtendón de Aquiles ${ }^{25}$ en nuestra serie, no hemos percibido esa complicación, ni hemos utilizado el abordaje transtendón.

Spennacchio y colaboradores ${ }^{26}$ en el 2016 realizaron una revisión sistemática de la bibliografía en relación con las artroscopías posteriores de tobillo, evaluando 46 artículos y 766 procedimientos, nueve artículos reportaron una tasa de complicaciones menores inferior al 7\% y de complicaciones mayores inferior al 2\%. En nuestra serie, las complicaciones observadas fueron todas menores y la mayoría de ellas con resolución a corto plazo.

Si bien se trata de un estudio retrospectivo, los datos se basaron en la historia clínica computarizada con seguimiento prospectivo de los pacientes, objetivando el examen y las complicaciones en cada consulta. Una debilidad es que, aunque todos los pacientes fueron sometidos a artroscopía posterior de tobillo y subastragalina, el motivo de la cirugía respondió a diferentes patologías, no se identificaron las complicaciones en referencia a los diagnósticos por los que fue tratado el paciente.

Creemos que, para evitar complicaciones, es necesario realizar el procedimiento en posición prona del paciente, permitiendo realizar portales paraaquilianos y manejar el instrumental con facilidad y comodidad, sin tracción de las partes blandas. También es necesario tener un conocimiento acabado de la anatomía artroscópica de la región posterior del tobillo y subtalar.

\section{Conclusiones}

La serie presentada ha mostrado que la artroscopía posterior del tobillo sin distracción por medio de los clásicos portales posterolateral y posteromedial es una técnica segura, con un bajo índice de complicaciones mayores y una baja morbilidad para el paciente, teniendo la ventaja de ser un método poco invasivo para resolver múltiples patologías posteriores de tobillo y retropié.

Referencias

1. Willits K, Sonneveld H, Amendola A, Giffin JR, Griffin S, Fowler PJ. Outcome of posterior ankle arthroscopy for hindfoot impingement. Arthroscopy. 2008; 24: 196-202. doi: 10.1016/j.arthro.2007.08.025.

2. Williams M, Ferkel R. Subtalar arthroscopy: indications, technics and results. Arthroscopy. 1998; 14(4): 373-81. Available in: https://doi. org/10.1016/S0749-8063(98)70004-0.

3. Amendola A, Lee KB, Saltzman CL, Suh JS. Technique and early experience with posterior arthroscopic subtalar arthrodesis. Foot Ankle Int. 2007; 28: 298-302.

4. Lee KB, Park CH, Seon JK, Kim MS. Arthroscopic subtalar arthrodesis using a posterior 2-portal approach in the prone position. Arthroscopy. 2010; 26: 230-8. doi: 10.1016/j.arthro.2009.07.008.

5. Golano P, Mariani PP, Rodríguez-Niedenfuhr M, Mariani PF, RuanoGil D. Arthroscopic anatomy of the posterior ankle ligaments. Arthroscopy. 2002; 18(4): 353-8.

6. Parisien JS. Arthroscopic treatment of ostheocondral lesions of the talus. Am J Sports Med. 1986; 68(6): 862-5. 
7. Sitler DF, Amendola A, Bailey CS, Thain LM, Spouge A. Posterior ankle arthroscopy: an anatomic study. J Bone Joint Surg Am. 2002; 84: 763-9.

8. Urguden M, Cevikol C, Dabak TK, Karaali K, Aydin AT, Apaydin A. Effect of joint motion on safety of portals in posterior ankle arthroscopy. Arthroscopy. 2009; 25: 1442-6. doi: 10.1016/j. arthro.2009.05.004.

9. Van Dijk CN, Scholten PE, Krips R. A 2-portal endoscopic approach for diagnosis and treatment of posterior ankle pathology. Arthroscopy. 2000; 16: 871-6. doi: 10.1053/jars.2000.19430.

10. Barber FA, Click J, Britt BT. Complications of ankle arthroscopy. Foot Ankle. 1990; 10(5): 263-6.

11. Carlson MJ, Ferkel RD. Complications in ankle and foot arthroscopy. Sports Med Arthrosc Rev. 2013; 21(2): 135-9. Available in: https://doi. org/10.1097/JSA.0b013e31828e5c6c).

12. Deng DF, Hamilton GA, Lee M, Rush S, Ford LA, Patel S. Complications associated with foot and ankle arthroscopy. J Foot Ankle Surg. 2011; 51(3): 281-4. https://doi.org/10.1053/j.jfas.2011.11.011.

13. Ferkel RD, Heath DD, Guhl JF. Neurological complications of ankle arthroscopy. Arthroscopy. 1996; 12(2): 200-8.

14. Ferkel RD, Small HN, Gittins JE. Complications in foot and ankle arthroscopy. Clin Orthop Relat Res. 2001; (391): 89-104. https://doi. org/10.1097/00003086-200110000-00010.

15. Yañez AJM, Rosales AFE, Lauritto D, Balmaceda MM, Amaya M, Yañez AS. Artroscopía anterior de tobillo. Complicaciones tempranas con técnica sin distracción. Rev Asoc Argent Ortop Traumatol. 2019; 84(3): 236-41. Available in: http://dx.doi.org/10.15417/issn.18527434.2019.84.3.885.

16. Gasparetto F, Collo G, Pisanu G, Villella D, Drocco L, Cerlon R, Bonasia DE. Posterior ankle and subtalar arthroscopy: indications, technique, and results. Curr Rev Musculoskelet Med. 2012; 5: 164-70. doi: 10.1007/s12178-012-9118-y.

17. Nickisch F, Barg A, Saltzman CL, Beals TC, Bonasia DE, Phisitkul P, et al. Postoperative complications in patients after posterior ankle and hindfoot arthroscopy. J Bone Joint Surg Am. 2012; 94: 439-46. doi: 10.2106/JBJS.K.00069.
18. Abramowitz Y, Wollstein R, Barzilay Y, London E, Matan Y, Shabat S, Nyska M. Outcome of resection of a symptomatic os trigonum. $J$ Bone Joint Surg Am. 2003; 85: 1051-7. doi: 10.2106/00004623-20030600000010.

19. Noguchi H, Ishii Y, Takeda M, Hasegawa A, Monden S, Takagishi K. Arthroscopic excision of posterior ankle bony impingement for early return to the field: short-term results. Foot Ankle Int. 2010; 31: 398403. https://doi.org/10.3113/FAI.2010.0398.

20. Webb J, Moorjani N, Radford M. Anatomy of the sural nerve and its relation to the Achilles tendon. Foot Ankle Int. 2000; 21: 475-7. doi: $10.1177 / 107110070002100604$.

21. Lee KB, Saltzman CL, Suh JS, Wasserman L, Amendola A. A posterior 3-portal arthroscopic approach for isolated subtalar arthrodesis. Arthroscopy. 2008; 24: 1306-10. doi: 10.1016/j.arthro.2006.01.025.

22. Keeling JJ, Guyton GP. Endoscopic flexor hallucis longus decompression: a cadaver study. Foot Ankle Int. 2007; 28: 810-4. Available in: https://doi.org/10.3113/FAI.2006.0810.

23. Phisitkul P, Amendola A, False FHL: a normal variant posing risks in posterior hindfoot endoscopy. Arthroscopy. 2010; 26: 714-8. doi: 10.1016/j.arthro.2009.07.026.

24. Carro LP, Golanó P, Vega J. Arthroscopic subtalar arthrodesis: the posterior approach in the prone position. Arthroscopy. 2007; 23: 445. e1-4. Available in: https://doi.org/10.1016/j.arthro.2006.07.049.

25. Horibe S, Kita K, Natsu-ume T, Hamada M, Mae T, Shino K. A novel technique of arthroscopic excision of a symptomatic os trigonum. Arthroscopy. 2008; 24: e1-4. Available in: doi.org/10.1016/j. arthro.2007.04.019.

26. Spennacchio P, Cucchi D, Randelli PS, van Dijk N. Evidence-based indications for hindfoot endoscopy. Knee Surg Sports Traumatol Arthrosc. 2016; 24(4): 1386-95. doi: 10.1007/s00167-015-3965-1.

Conflicto de intereses: Los autores no declaran conflicto de intereses. 